hep-th/0503082

\title{
Annulus Amplitudes in the Minimal Superstring
}

\author{
Kazumi Okuyama \\ Department of Physics and Astronomy, University of British Columbia \\ Vancouver, BC, V6T 1Z1, Canada \\ kazumi@physics.ubc.ca
}

\begin{abstract}
We study the annulus amplitudes in the $(2,4)$ minimal superstring theory using the continuum worldsheet approach. Our results reproduce the semiclassical behavior of the wavefunctions of FZZT-branes recently studied in hep-th/0412315 using the dual matrix model. We also study the multi-point functions of neutral FZZT-branes and find the agreement between their semiclassical limit and the worldsheet annulus calculation.
\end{abstract}

March, 2005 


\section{Introduction}

Minimal superstring theories are interesting arena to study various aspects of string theory [1 [8]. The existence of dual matrix models allows us to study nonperturbative phenomena in a very controlled setup. In particular, we can expect to understand the physics of D-branes in a quantum regime and the open-closed duality in a very precise way.

In [9] it is realized that the FZZT-brane is a useful probe of the target space in minimal string theories. In the matrix model description, the FZZT-brane corresponds to the determinant operator $\operatorname{det}(x-M)$ and one can define the wavefunction $\psi(x)$ of FZZT-brane as a double scaling limit of the expectation value $\langle\operatorname{det}(x-M)\rangle$. Due to the Stokes' phenomenon, the semiclassical moduli space of FZZT-brane, which is represented by a Riemann surface, is replaced by the entire complex $x$-plane in the full theory, and correspondingly $\psi(x)$ is an entire function of $x$. In particular, at a topological point the FZZT wavefunction is given by a (generalized) Airy function [9, [10,11] which is indeed entire. This implies that a semiclassical picture of target space measured by the FZZTbrane is completely different from the picture in the full nonperturbative theory.

One would expect that the physics of FZZT-branes and their wavefunctions in minimal superstring theories is much richer than that of their bosonic cousins, given the fact that there is an interesting interplay between RR fluxes and D-branes in minimal superstring theories. In a recent paper [12], the simplest minimal superstring, i.e., the $(p, q)=(2,4)$ theory has been studied. This theory contains two types of FZZT-branes, the branes which are either neutral or charged under the RR potential. The neutral brane is easy to describe in the 0A language (complex matrix model), while the charged brane is naturally described in the $0 \mathrm{~B}$ language (hermitian matrix model). It is argued [1] that $0 \mathrm{~A}$ and $0 \mathrm{~B}$ theories are actually equivalent for the $(p, q)=(2,4)$ case.

In this paper, we will present a one more nontrivial check for the duality between the matrix model and the $(2,4)$ minimal superstring theory. We will study the annulus amplitudes in the $(2,4)$ theory using the worldsheet approach and find complete agreement with the semiclassical behavior of the wavefunctions of FZZT-branes obtained in [12]. Although the wavefunctions are analytic in the bulk cosmological constant $\mu$, the semiclassical limit is dependent on the sign of $\mu$, so we need to study the two cases $\mu>0$ and $\mu<0$ separately.

The semiclassical expansion of FZZT wavefunction involves only the annulus between the FZZT-brane and itself. On the other hand, our worldsheet calculation gives more general amplitudes between two FZZT-branes with different boundary cosmological constants 
$\mu_{B} \neq \mu_{B}^{\prime}$. These amplitudes naturally appear in the semiclassical limit of multi-point functions of FZZT-branes. We will study the multi-brane correlators of neutral FZZTbranes using the complex matrix model and again find an impressive agreement with the worldsheet calculation.

This paper is organized as follows. In section 2, we study the annulus amplitudes in the $\mu>0$ phase and compare them with the semiclassical behavior of the wavefunctions of FZZT-branes. In section 3, we repeat the analysis for the $\mu<0$ phase. In section 4 , we consider the multi-point functions of neutral FZZT-branes and show that the semiclassical behavior of those correlators is reproduced from the worldsheet annulus computation. Appendix A is devoted to the detailed calculation of the amplitudes.

\section{One-Cut Phase $(\mu>0)$}

Let us first summarize the semiclassical behaviors of the wavefunctions of FZZT branes obtained in [12]. In this section, we will consider the case $\mu>0$, or the one-cut phase in the type $0 \mathrm{~B}$ picture. In general, we expect that in the semiclassical regime $(\mu \rightarrow+\infty$, $x \rightarrow \pm i \infty$ with $x / \sqrt{\mu}$ fixed) the wavefunction of FZZT-brane behaves as

$$
\psi(x, q) \sim \exp \left[D(x)+\frac{1}{2} Z(x, x)+|q| \widetilde{Z}(x, \epsilon)+\cdots\right]
$$

where $x$ is related to the boundary cosmological constant $\mu_{B}$ by $x=i \mu_{B}$. The first term $D(x)$ in (2.1) denotes the disk amplitude. The explicit form of disk for the charged FZZT-brane $D_{ \pm}(x)$ and for the neutral FZZT-brane $D_{0}(x)$ reads

$$
D_{ \pm}(x)=\left\{\begin{array}{ll}
-i\left(\frac{4}{3} x^{3}+\mu x\right) \\
-i \frac{4}{3}\left(x^{2}+\frac{\mu}{2}\right)^{\frac{3}{2}}
\end{array} \quad, \quad D_{0}(x)=\left\{\begin{array}{ll}
-\frac{4}{3} \sqrt{2}\left(\frac{\mu}{2}-x^{2}\right)^{\frac{3}{2}} & \mu>0 \\
-i \sqrt{2}\left(\frac{4}{3} x^{3}-\mu x\right) & \mu<0
\end{array} .\right.\right.
$$

The second term $Z(x, x)$ in $(2.1)$ is the annulus between the FZZT-brane and itself. The factor of $1 / 2$ in front of $Z(x, x)$ comes from the fact that the open strings are ending on the

same brane [9]. The third term $|q| \widetilde{Z}(x, \epsilon)$ comes from the interaction between the FZZTbrane and the $(1,1)$ ZZ-branes in the background. At the leading order, this interaction is represented by the annulus between the FZZT-brane and a single ZZ-brane with charge $\epsilon=\operatorname{sign}(q)$, multiplied by the number $|q|$ of ZZ-branes. 
It is shown in [12] that the semiclassical wavefunction of the neutral FZZT-brane is given by

$$
\psi_{0}(x, q) \sim(-i x)^{-|q|} \exp \left[D_{0}(x)-\frac{1}{4} \log \left(\mu-2 x^{2}\right)+\frac{|q|}{2} \log \left(\frac{\sqrt{\mu-2 x^{2}}-\sqrt{\mu}}{\sqrt{\mu-2 x^{2}}+\sqrt{\mu}}\right)+\cdots\right]
$$

and the wavefunction of the charged FZZT-brane behaves as

$$
\begin{array}{ll}
\psi_{+}(x, q) \sim \exp \left[D_{+}(x)+q \log \left\{-i 2 \sqrt{2}\left(x-x_{Z Z}\right)\right\}+\cdots\right] & \operatorname{Im} x>0 \\
\psi_{-}(x, q) \sim \exp \left[D_{-}(x)-q \log \left\{+i 2 \sqrt{2}\left(x-x_{Z Z}\right)\right\}+\cdots\right] & \operatorname{Im} x<0,
\end{array}
$$

where $x_{Z Z}=\mp i \frac{\sqrt{\mu}}{2}$ is the value of $x$ associated with the $(1,1)$ ZZ-brane, which corresponds to the extrema of the effective potential in the dual matrix model picture. For the neutral brane wavefunction (2.3), we have factored out $x^{-|q|}$ as an overall factor. As discussed in [12], this is necessary in the $\mu<0$ phase when comparing $\psi_{0}$ to the worldsheet calculation. Here we followed the same prescription also for the $\mu>0$ phase in order to identify the $q$-dependent term $|q| \widetilde{Z}(x, \epsilon)$ correctly. In other words, the rescaled wavefunction $\widetilde{\psi}_{0}(x)=$ $(-i x)^{|q|} \psi_{0}(x)$ is the one which shows the expected semiclassical behavior (2.1).

In the rest of this section, we will reproduce these expressions from the continuum worldsheet calculation.

\subsection{Worldsheet Computation of the Annulus between FZZT Branes}

In the worldsheet approach, D-branes are described by using boundary states. The boundary states of the charged and neutral FZZT-branes are given by [ , 3, 12 ]

$$
\begin{aligned}
&|\sigma, \xi, \eta=-1\rangle=\int_{0}^{\infty} d P\left(\frac{\mu}{2}\right)^{\frac{i P}{b}}\left(\cos (\pi P \sigma) A_{N S}(P)|P, \eta=-1\rangle_{N S}\right. \\
&\left.+\xi \cos (\pi P \sigma) A_{R}(P)|P, \eta=-1\rangle_{R}\right) \\
&|\sigma, 0, \eta=+1\rangle=\sqrt{2} \int_{0}^{\infty} d P\left(\frac{\mu}{2}\right)^{\frac{i P}{b}} \cos (\pi P \sigma) A_{N S}(P)|P, \eta=+1\rangle_{N S}
\end{aligned}
$$

where $b=\frac{1}{\sqrt{2}}$ and $A_{N S}(P), A_{R}(P)$ are the wavefunctions of $\mathcal{N}=1$ super-Liouville theory 13,14

$$
A_{N S}(P)=\frac{\Gamma(1-i P b) \Gamma\left(1-i P b^{-1}\right)}{-\sqrt{2} \pi i P}, \quad A_{R}(P)=\frac{1}{\pi b^{2}} \Gamma\left(\frac{1}{2}-i P b\right) \Gamma\left(\frac{1}{2}-i P b^{-1}\right) .
$$

${ }^{1}$ In this paper, we use the normalization of $P$ and $\sigma$ in [3], while the convention of $\mu$ is that of [12]. 
In (2.5), $\xi= \pm 1$ represents the charge of the brane and $\eta= \pm 1$ specifies the boundary condition of the supercharge $Q=i \eta \bar{Q}$.

Now let us consider the annulus amplitudes between FZZT-branes

$$
\begin{aligned}
& Z\left(\sigma, 0 \mid \sigma^{\prime}, 0\right)=\int_{0}^{\infty} d t_{c}\left\langle\sigma, 0, \eta=+1\left|q^{\frac{1}{2}\left(L_{0}+\widetilde{L}_{0}\right)}\right| \sigma^{\prime}, 0, \eta=+1\right\rangle \\
& Z\left(\sigma, \xi \mid \sigma^{\prime}, \xi\right)=\int_{0}^{\infty} d t_{c}\left\langle\sigma, \xi, \eta=-1\left|q^{\frac{1}{2}\left(L_{0}+\widetilde{L}_{0}\right)}\right| \sigma^{\prime}, \xi, \eta=-1\right\rangle \\
& Z\left(\sigma, 0 \mid \sigma^{\prime}, \xi\right)=-\int_{0}^{\infty} d t_{c}\left\langle\sigma, 0, \eta=+1\left|q^{\frac{1}{2}\left(L_{0}+\widetilde{L}_{0}\right)}\right| \sigma^{\prime}, \xi, \eta=-1\right\rangle
\end{aligned}
$$

where $q=e^{-2 \pi t_{c}}$. In the last equation in (2.7), we have put an extra minus sign for the amplitude $Z\left(\sigma, 0 \mid \sigma^{\prime}, \xi\right)$, since the modes running in the open string channel are in the Rsector and hence fermionic. From the calculations in appendix A, the annulus amplitudes between various FZZT-branes in the $\mu>0$ phase are found to be

$$
\begin{aligned}
& Z\left(\sigma, 0 \mid \sigma^{\prime}, 0\right)=-\log \left(2 \sqrt{\mu} \cosh \theta+2 \sqrt{\mu} \cosh \theta^{\prime}\right) \\
& Z\left(\sigma, \xi \mid \sigma^{\prime}, \xi^{\prime}\right)=\frac{-1+\xi \xi^{\prime}}{2} \log \left(2 \sqrt{\mu} \cosh \theta+2 \sqrt{\mu} \cosh \theta^{\prime}\right) \\
& Z\left(\sigma, 0 \mid \sigma^{\prime}, \xi\right)=\frac{1}{2} \log \left(2 \sqrt{\mu} \cosh \left(\theta+\theta^{\prime}\right)+\sqrt{2 \mu}\right)+\frac{1}{2} \log \left(2 \sqrt{\mu} \cosh \left(\theta-\theta^{\prime}\right)+\sqrt{2 \mu}\right)
\end{aligned}
$$

where we defined $\theta$ by

$$
\theta=\frac{\pi b \sigma}{2}=\frac{\pi \sigma}{\sqrt{8}}
$$

\section{2. $Z(x, x)$}

Now we can compare the worldsheet result (2.8) with the matrix model result (2.3), (2.4). Let us consider the term $Z(x, x)$ in the semiclassical wavefunction. For the charged branes, the worldsheet result (2.8) shows that the annulus between FZZT-branes carrying the same charge vanishes

$$
Z\left(\sigma, \xi \mid \sigma^{\prime}, \xi\right)=0
$$

This agrees with the absence of the $q$-independent term in the wavefunctions (2.4).

For the neutral brane, recalling that $\theta$ and $x$ are related by $x=i \sqrt{\frac{\mu}{2}} \sinh \theta$, we see that the worldsheet result (2.8) and the matrix model result (2.3) agree with each other up to an irrelevant additive constant

$$
Z(\sigma, 0 \mid \sigma, 0)=-\log (4 \sqrt{\mu} \cosh \theta)=-\frac{1}{2} \log \left(\mu-2 x^{2}\right)-\log 4
$$




\section{3. $\widetilde{Z}(x, \epsilon)$}

Next consider the term $\widetilde{Z}(x, q)$. The annulus between a FZZT-brane and a ZZ-brane can be obtained from the FZZT-FZZT annulus by writing the ZZ boundary state as a linear combination of the FZZT boundary states [3, 15]

$$
|(1,1), \xi\rangle=\left|\sigma_{1,1}, \xi\right\rangle-\left|\sigma_{1,-1},-\xi\right\rangle
$$

where $\sigma_{m, n}=i\left(m b^{-1}+n b\right)$. From this relation (2.12) and the amplitude $Z\left(\sigma, 0 \mid \sigma^{\prime}, \xi\right)$ in (2.8), the annulus between a neutral FZZT-brane and a $(1,1)$ ZZ-brane is obtained as

$$
Z(\sigma, 0 \mid(1,1), \xi)=Z\left(\sigma, 0 \mid \sigma_{1,1}, \xi\right)-Z\left(\sigma, 0 \mid \sigma_{1,-1},-\xi\right)=\frac{1}{2} \log \left(\frac{\cosh \theta-1}{\cosh \theta+1}\right)
$$

This is independent of the charge $\xi$ of the ZZ-brane since only the NSNS exchange in the closed string channel contributes to this amplitude. From the relation $x=i \sqrt{\frac{\mu}{2}} \sinh \theta$, one can see that (2.13) agrees with the $|q|$ dependent term inside the exponential of (2.3). Note that it is necessary to factor out $x^{-|q|}$ for this agreement to work.

Similarly, the annulus between a charged FZZT-brane and a $(1,1)$ ZZ-brane is given by

$$
\begin{aligned}
Z\left(\sigma, \xi \mid(1,1), \xi^{\prime}\right) & =Z\left(\sigma, \xi \mid \sigma_{1,1}, \xi^{\prime}\right)-Z\left(\sigma, \xi \mid \sigma_{1,-1},-\xi^{\prime}\right) \\
& =\frac{\xi \xi^{\prime}-1}{2} \log \left[2 \sqrt{\mu}\left(\cosh \theta-\frac{1}{\sqrt{2}}\right)\right]+\frac{\xi \xi^{\prime}+1}{2} \log \left[2 \sqrt{\mu}\left(\cosh \theta+\frac{1}{\sqrt{2}}\right)\right] .
\end{aligned}
$$

Since $\xi \xi^{\prime}= \pm 1$, 2.14 can be written more compactly as

$$
Z\left(\sigma, \xi \mid(1,1), \xi^{\prime}\right)=\xi \xi^{\prime} \log \left[2 \sqrt{\mu}\left(\cosh \theta+\frac{\xi \xi^{\prime}}{\sqrt{2}}\right)\right]
$$

Using the relation $x=i \xi \sqrt{\frac{\mu}{2}} \cosh \theta$ and $x_{Z Z}=-i \xi^{\prime} \frac{\sqrt{\mu}}{2}$, (2.15) is further rewritten as

$$
|q| Z\left(\sigma, \xi \mid(1,1), \xi^{\prime}\right)=2 q_{b} q \log \left[-i 2 \sqrt{2} \xi\left(x-x_{Z Z}\right)\right]
$$

where we identified $\xi=2 q_{b}=\operatorname{sign}(\operatorname{Im} x)$ and $\xi^{\prime}=\operatorname{sign}(q)$. This reproduces the $q$-dependent term in the charged FZZT-brane wavefunctions (2.4). Although the final result is proportional to the charge of ZZ-brane, this amplitude receives contributions both from the RR sector and the NSNS sector. However, there is a qualitative difference between the RR 
and the NSNS contributions for this amplitude. In the NSNS sector the pole of $\left|A_{N S}(P)\right|^{2}$ is canceled by a factor coming from the ZZ boundary state

$$
\left(\cos \pi P \sigma_{1,1}-\cos \pi P \sigma_{1,-1}\right)\left|A_{N S}(P)\right|^{2}=1 .
$$

Therefore, there is no pole at $P=0$ in the integral representation of this NSNS contribution and the result is finite:

$$
Z\left(\sigma, \xi \mid(1,1), \xi^{\prime}\right)_{N S}=\frac{1}{2} \int_{-\infty}^{\infty} \frac{d P}{P} \frac{\sinh \frac{\pi P}{\sqrt{2}}}{\cosh \sqrt{2} \pi P} \cos (\pi P \sigma) .
$$

On the other hand, the corresponding relation for the RR sector

$$
\left(\cos \pi P \sigma_{1,1}+\cos \pi P \sigma_{1,-1}\right)\left|A_{R}(P)\right|^{2}=1
$$

does not remove the pole at $P=0$ :

$$
Z\left(\sigma, \xi \mid(1,1), \xi^{\prime}\right)_{R}=-\frac{\xi \xi^{\prime}}{2} \int_{-\infty}^{\infty} \frac{d P}{P} \frac{\cosh \frac{\pi P}{\sqrt{2}}}{\sinh \sqrt{2} \pi P} \cos (\pi P \sigma)
$$

Therefore, the RR contribution contains a divergence proportional to the volume of Liouville direction.

Some comments on the statistics of open strings between FZZT and ZZ branes are in order. In the amplitude $Z(\sigma, 0 \mid(1,1), \xi)$, the open string running along the loop is in the $\mathrm{R}$ sector. This implies that the open string between the $(1,1) \mathrm{ZZ}$-brane and the neutral FZZT-brane is fermionic. This is consistent with the fact that the neutral brane is represented by a determinant in the complex matrix model [12]. On the other hand, $Z\left(\sigma, \xi \mid(1,1), \xi^{\prime}\right)$ is in the NS sector in the open string channel and hence the open string between the $(1,1)$ ZZ-brane and the charged FZZT-brane is bosonic.

This is in contrast with the situation in the bosonic minimal string. In the bosonic theory, the FZZT-brane corresponds to the determinant in the matrix model and consequently the open strings between FZZT and ZZ branes are fermionic, despite the fact that the theory itself is a bosonic string theory. In the worldsheet approach, this fermionic nature of open string can be taken care of by adding an extra minus sign relative to the relation between the FZZT boundary state and the ZZ boundary state in the Liouville theory alone 16]

$$
|m, n\rangle=-\left|\sigma_{m, n}\right\rangle+\left|\sigma_{m,-n}\right\rangle
$$


However, in the case of minimal superstring, this modification does not lead to a consistent picture because the open string between ZZ and FZZT can be either bosonic or fermionic depending on the charge of FZZT-branes in question, as we saw above. Instead, we keep the relation (2.12) intact and add an extra minus sign to the annulus $Z\left(\sigma, 0 \mid \sigma^{\prime}, \xi\right)$ in order to represent the fermionic nature of the modes running along the open string one-loop. Our choice (2.12) of the relative sign between ZZ and FZZT boundary states is justified by the fact that the disk amplitude of the ZZ-brane is negative with this choice of relative $\operatorname{sign}$ 日

$$
D_{ \pm}^{\mathrm{ZZ}}=D_{ \pm}\left(\sigma_{1,1}\right)-D_{\mp}\left(\sigma_{1,-1}\right)=-\frac{2}{3} \mu^{\frac{3}{2}}<0 .
$$

This sign guarantees that the nonperturbative effect associated with an instanton-antiinstanton pair, which goes like $e^{D_{+}^{\mathrm{ZZ}}+D_{-}^{\mathrm{ZZ}}} \sim e^{-\frac{4}{3} \mu^{\frac{3}{2}}}$, is exponentially small in the weak coupling regime $g_{s} \sim \mu^{-3 / 2} \ll 1$ [16].

\section{Two-Cut Phase $(\mu<0)$}

The computation in the $\mu<0$ phase is almost similar to the $\mu>0$ case. The FZZT boundary states in this phase are given by [3], 12]

$$
\begin{gathered}
|\sigma, \xi, \eta=-1\rangle^{\text {naive }}=\int_{0}^{\infty} d P\left(-\frac{\mu}{2}\right)^{\frac{i P}{b}}\left(\cos (\pi P \sigma) A_{N S}(P)|P, \eta=-1\rangle_{N S}\right. \\
\left.-i \xi \sin (\pi P \sigma) A_{R}(P)|P, \eta=-1\rangle_{R}\right) \\
|\sigma, 0, \eta=+1\rangle=\sqrt{2} \int_{0}^{\infty} d P\left(-\frac{\mu}{2}\right)^{\frac{i P}{b}} \cos (\pi P \sigma) A_{N S}(P)|P, \eta=+1\rangle_{N S} .
\end{gathered}
$$

It was pointed out in [12] that for the charged FZZT-brane the coupling to the zeromomentum RR potential should be added to the naive boundary state

$$
|\sigma, \xi, \eta=-1\rangle=|\sigma, \xi, \eta=-1\rangle^{\text {naive }}+\frac{\xi}{2} V_{R}|0\rangle \text {. }
$$

The annulus amplitudes $Z\left(\sigma, 0 \mid \sigma^{\prime}, 0\right)$ and $Z\left(\sigma, 0 \mid \sigma^{\prime}, \xi\right)$ involving neutral branes are obtained from the $\mu>0$ result by simply sending $\mu \rightarrow-\mu$, since the RR sector does not contribute to those amplitudes:

$$
\begin{aligned}
Z\left(\sigma, 0 \mid \sigma^{\prime}, 0\right) & =-\log \left(2 \sqrt{-\mu} \cosh \theta+2 \sqrt{-\mu} \cosh \theta^{\prime}\right) \\
Z\left(\sigma, 0 \mid \sigma^{\prime}, \xi\right) & =\frac{1}{2} \log \left(2 \sqrt{-\mu} \cosh \left(\theta+\theta^{\prime}\right)+\sqrt{-2 \mu}\right) \\
& +\frac{1}{2} \log \left(2 \sqrt{-\mu} \cosh \left(\theta-\theta^{\prime}\right)+\sqrt{-2 \mu}\right) .
\end{aligned}
$$

2 We would like to thank N. Seiberg and D. Shih for this argument. 
Let us compare (3.3) with the semiclassical wavefunction of neutral FZZT brane

$$
\psi_{0}(x, q) \sim(-i x)^{-|q|} \exp \left[D_{0}(x)-\frac{1}{2} \log x+\cdots\right] .
$$

Since there are no charged ZZ-branes in this phase and the neutral brane has no RR Ishibashi component in its boundary state, the leading semiclassical terms (i.e. disk and annulus) should be $q$-independent [12]. Therefore, the rescaled wavefunction $(-i x)^{|q|} \psi_{0}(x)$, which is independent of $q$ at the leading order, is the natural object to compare with the worldsheet calculation. Using the relation $x=i \sqrt{-\frac{\mu}{2}} \cosh \theta$, one can see that $Z(\sigma, 0 \mid \sigma, 0)$ in (3.3) agrees with the $-\log x$ term in (3.4).

In a similar manner as in appendix $\mathrm{A}$, the annulus amplitude between the charged branes using the naive boundary state is evaluated as

$$
\begin{aligned}
Z\left(\sigma, \xi \mid \sigma^{\prime}, \xi^{\prime}\right)^{n a i v e} & =\frac{1}{2} \int_{-\infty}^{\infty} d P \frac{P}{P^{2}+\varepsilon^{2}} \frac{\cos \left(\pi P \sigma+\xi \xi^{\prime} \pi P \sigma^{\prime}\right)}{\sinh (\sqrt{8} \pi P)} \\
& =\frac{1}{2 \sqrt{8} \varepsilon}-\log \left[2 \cosh \left(\frac{\theta+\xi \xi^{\prime} \theta^{\prime}}{2}\right)\right] \\
& =-\frac{1}{4} \log (-\mu)-\log \left[2 \cosh \left(\frac{\theta+\xi \xi^{\prime} \theta^{\prime}}{2}\right)\right]+\text { const } .
\end{aligned}
$$

From this expression, the amplitude between a charged FZZT-brane and itself is found to be

$$
Z(\sigma, \xi \mid \sigma, \xi)^{\text {naive }}=-\frac{1}{4} \log (-\mu)-\log (2 \cosh \theta)
$$

Now let us compare the amplitude (3.6) with the semiclassical wavefunction of charged FZZT-brane [12]

$$
\psi_{+} \sim \exp \left[-\frac{4}{3}\left(-\frac{\mu}{2}\right)^{\frac{3}{2}} \cosh ^{3} \theta-\frac{1}{2} \log (2 \cosh \theta)+\frac{1}{2} \theta+q\left(\theta+\frac{1}{2} \log (-\mu)\right)\right] .
$$

It is argued [12 that the annulus amplitude extracted from the asymptotic wavefunction (3.7) has a decomposition

$$
Z(\sigma \mid \sigma)=-\log (2 \cosh \theta)+\theta=Z(\sigma \mid \sigma)^{\text {naive }}+\partial_{q} D^{\text {naive }}(\sigma)-\frac{1}{4} \partial_{q}^{2} F
$$

where $Z(\sigma \mid \sigma)^{\text {naive }}$ is the naive annulus amplitude, $\partial_{q} D^{\text {naive }}(\sigma)=\theta$ is the disk one-point function of $V_{R}$, and $\partial_{q}^{2} F=-\log (-\mu)$ is the two-point function of $V_{R}$ on the sphere. Therefore, the matrix model result predicts that [12]

$$
Z(\sigma \mid \sigma)^{\text {naive }}=-\frac{1}{4} \log (-\mu)-\log (2 \cosh \theta)
$$


Clearly, our boundary state computation (3.6) for the naive part of annulus amplitude agrees with the matrix model result (3.9) as conjectured in [12].

To summarize, our analysis of annulus amplitudes in section 2 and 3 precisely reproduces the all known asymptotic behaviors of semiclassical wavefunctions studied in [12]. This agreement between the worldsheet computation and the matrix model result can be thought of as strong evidence of the duality between the $(2,4)$ minimal superstring and the double scaled matrix model in the presence of D-branes.

\section{Multi-Point Correlators of Neutral Branes}

As we saw above, only the annulus amplitude $Z(x, x)$ between a FZZT-brane and itself appears in the semiclassical expansion of the wavefunctions, or the one-point functions of the FZZT operators. It is clear that the natural place where the annulus amplitudes $Z\left(x, x^{\prime}\right)$ with $x \neq x^{\prime}$ show up is the multi-point functions of FZZT-brane operators. For the neutral branes, as we will see below, it is straightforward to generalize the one-point function $\left\langle B_{0}(x)\right\rangle$ to the multi-point functions $\left\langle\prod_{i} B_{0}\left(x_{i}\right)\right\rangle$ as in the case of bosonic minimal string [9, 10]. On the other hand, the calculation of charged brane correlators turned out to be not as straightforward as that of neutral branes. Therefore, in this paper we will consider only the correlators of neutral branes and leave the matrix model calculation of charged brane correlators as an interesting open problem.

It is argued [12] that the wavefunction of neutral FZZT brane is obtained as a double scaling limit of the determinant operator in a complex matrix model

$$
\psi_{0}(x)=\lim _{N \rightarrow \infty} \frac{1}{\sqrt{h_{N}}} e^{-V\left(x^{2}\right)}\left\langle\operatorname{det}\left(x^{2}-M M^{\dagger}\right)\right\rangle=\frac{1}{Z}\left\langle B_{0}(x)\right\rangle .
$$

We can naturally promote this equation as the definition of the operator $B_{0}(x)$

$$
B_{0}(x)=\lim _{N \rightarrow \infty} \frac{1}{\sqrt{h_{N}}} e^{-V\left(x^{2}\right)} \operatorname{det}\left(x^{2}-M M^{\dagger}\right) .
$$

As explained in the appendix A.3 of [12], we can apply the determinant formula of [17] to evaluate the correlator of determinants in the complex matrix model

$$
\left\langle\prod_{i} \operatorname{det}\left(x_{i}^{2}-M M^{\dagger}\right)\right\rangle=\frac{\operatorname{det}\left(P_{N+j-1}\left(x_{i}^{2}\right)\right)}{\Delta\left(x^{2}\right)},
$$


where $\Delta(\lambda)=\prod_{i>j}\left(\lambda_{i}-\lambda_{j}\right)$ is the Vandermonde determinant and $P_{n}(\lambda)$ are orthogonal polynomials. In the double scaling limit, the shift in the index of the orthogonal polynomials becomes a derivative with respect to $\mu$. Therefore, after double scaling limit (4.3) becomes

$$
\frac{1}{Z}\left\langle\prod_{i} B_{0}\left(x_{i}\right)\right\rangle=\frac{\operatorname{det}_{i j}\left(\partial_{\mu}^{j-1} \psi_{0}\left(x_{i}, \mu\right)\right)}{\Delta\left(x^{2}\right)} .
$$

Note that a similar expression for the multi-brane correlator in bosonic minimal string theory was obtained in [9, 10].

From (4.4), one can see that the semiclassical expansion of the two neutral FZZTbrane correlator has a prefactor

$$
\frac{\partial_{\mu} D_{0}(x)-\partial_{\mu} D_{0}\left(x^{\prime}\right)}{x^{2}-x^{\prime 2}}=\frac{2}{\sqrt{|\mu|}\left(\cosh \theta+\cosh \theta^{\prime}\right)} .
$$

Note that this expression is valid for both signs of $\mu$. As expected, 4.5) exactly coincides (up to an irrelevant numerical factor) with the exponential of the annulus amplitude between two neutral branes $Z\left(\sigma, 0 \mid \sigma^{\prime}, 0\right)$ given in (2.7) and (3.3). This gives another nontrivial check for the duality between the matrix model and the minimal superstring, in the multi-brane sector in this case.

As we mentioned above, it would be very interesting to generalize the one-point functions $\left\langle B_{ \pm}(x)\right\rangle$ of charged FZZT-brane to the multi-point functions $\left\langle\prod_{i} B_{ \pm}\left(x_{i}\right)\right\rangle$ and study their interplay with the background RR flux.

Acknowledgments: I would like to thank Nathan Seiberg and David Shih for useful discussions and encouragement. I would also like to thank Jongwon Park for discussions.

\section{Appendix A. Computation of Annulus Amplitudes}

In this appendix, we will present details of the calculation of annulus amplitudes (2.8) and (3.3) in the text. See [15,16] for similar calculations in the bosonic minimal string.

Before going into details, let us summarize some useful formulas used in the following computation. We need the absolute value of the super-Liouville wavefunctions $A_{N S}(P), A_{R}(P)$ given in (2.6):

$$
\left|A_{N S}(P)\right|^{2}=\frac{1}{2 \sinh \pi P b \sinh \pi P b^{-1}}, \quad\left|A_{R}(P)\right|^{2}=\frac{1}{2 \cosh \pi P b \cosh \pi P b^{-1}} .
$$


We define the partition functions as

$$
\begin{gathered}
\eta(q)=q^{\frac{1}{24}} \prod_{n=1}^{\infty}\left(1-q^{n}\right) \\
f_{R}(q)=\sqrt{2} q^{\frac{1}{24}} \prod_{n=1}^{\infty}\left(1+q^{n}\right) \\
f_{N S}(q)=q^{-\frac{1}{48}} \prod_{n=1}^{\infty}\left(1+q^{n-\frac{1}{2}}\right) \\
f_{\widetilde{N S}}(q)=q^{-\frac{1}{48}} \prod_{n=1}^{\infty}\left(1-q^{n-\frac{1}{2}}\right)
\end{gathered}
$$

and the following expansions for the ratio of $\eta(q)$ and $f(q)$ 's are useful:

$$
\begin{aligned}
& \frac{\eta(q)}{f_{N S}(q)}=\frac{1}{2} \sum_{n \in \mathbb{Z}}(-1)^{\frac{1}{2} n(n+1)} q^{\frac{1}{4}\left(n+\frac{1}{2}\right)^{2}}=\sum_{k \in \mathbb{Z}}\left[q^{\frac{(8 k+1)^{2}}{16}}-q^{\frac{(8 k+3)^{2}}{16}}\right] \\
& \frac{\eta(q)}{f_{R}(q)}=\frac{1}{\sqrt{2}} \sum_{n \in \mathbb{Z}}(-1)^{n} q^{n^{2}} \\
& \frac{\eta(q)}{f_{\widetilde{N S}}(q)}=\frac{1}{2} \sum_{n \in \mathbb{Z}} q^{\frac{1}{4}\left(n+\frac{1}{2}\right)^{2}}=\sum_{k \in \mathbb{Z}}\left[q^{\frac{(8 k+1)^{2}}{16}}+q^{\frac{(8 k+3)^{2}}{16}}\right] .
\end{aligned}
$$

(A.3) can be easily shown by using Jacobi triple product identity. We will also use the following summation formulas

$$
\begin{aligned}
\sum_{k \in \mathbb{Z}} \frac{1}{x^{2}+(k+a)^{2}} & =\frac{\pi}{x} \cdot \frac{\sinh (2 \pi x)}{\cosh (2 \pi x)-\cos (2 \pi a)} \\
\sum_{n \in \mathbb{Z}} \frac{(-1)^{n}}{n^{2}+a^{2}} & =\frac{\pi}{a \sinh (\pi a)} .
\end{aligned}
$$

\section{A.1. Neutral-Neutral Amplitude: $Z\left(\sigma, 0 \mid \sigma^{\prime}, 0\right)$}

Let us start with the annulus amplitude between two neutral branes described by the boundary state $|\sigma, 0, \eta=+\rangle$ in $(2.5)$ :

$$
Z\left(\sigma, 0 \mid \sigma^{\prime}, 0\right)=2 \int_{0}^{\infty} d P\left|A_{N S}(P)\right|^{2} \cos (\pi P \sigma) \cos \left(\pi P \sigma^{\prime}\right) \int_{0}^{\infty} d t_{c}\left[\frac{\eta(q)}{f_{N S}(q)}\right]^{2} \frac{f_{N S}(q)}{\eta(q)} q^{\frac{P^{2}}{2}}
$$

Here, the factor $\left[\frac{\eta}{f_{N S}}\right]^{2}$ is the ghost contribution and $\frac{f_{N S}(q)}{\eta(q)} q^{\frac{P^{2}}{2}}$ is the NS character of $\mathcal{N}=1$ super-Liouville theory. 
Using the expansion (A.3) and the summation formula (A.4), the $t_{c}$ integral becomes $\left(\right.$ recall $\left.q=e^{-2 \pi t_{c}}\right)$

$$
\int_{0}^{\infty} d t_{c} \frac{\eta(q)}{f_{N S}(q)} q^{\frac{P^{2}}{2}}=\frac{1}{\pi} \sum_{k \in \mathbb{Z}}\left[\frac{1}{P^{2}+\frac{(8 k+1)^{2}}{8}}-\frac{1}{P^{2}+\frac{(8 k+3)^{2}}{8}}\right]=\frac{\sinh \frac{\pi P}{\sqrt{2}}}{P \cosh (\sqrt{2} \pi P)} .
$$

Then the amplitude (A.5) becomes

$$
\begin{aligned}
Z\left(\sigma, 0 \mid \sigma^{\prime}, 0\right) & =\int_{-\infty}^{\infty} d P\left|A_{N S}(P)\right|^{2} \cos (\pi P \sigma) \cos \left(\pi P \sigma^{\prime}\right) \frac{\sinh \frac{\pi P}{\sqrt{2}}}{P \cosh (\sqrt{2} \pi P)} \\
& =\int_{-\infty}^{\infty} \frac{d P}{P} \frac{\cos (\pi P \sigma) \cos \left(\pi P \sigma^{\prime}\right)}{\sinh (\sqrt{8} \pi P)}
\end{aligned}
$$

As discussed in [16], the double pole at $P=0$ can be regularized by replacing

$$
\frac{1}{P} \Rightarrow \lim _{\varepsilon \rightarrow+0} \frac{P}{P^{2}+\varepsilon^{2}} .
$$

By closing the contour of the $P$-integral and picking up the residues of the poles, the integral (A.7) is evaluated as

$$
Z\left(\sigma, 0 \mid \sigma^{\prime}, 0\right)=\frac{1}{\sqrt{8} \varepsilon}-\log \left(2 \cosh \theta+2 \cosh \theta^{\prime}\right) .
$$

We identify the divergence $1 / \varepsilon$ as the volume of the Liouville direction. Recalling our definition of the vertex operator $\mathcal{V}_{P}=e^{\left(\frac{Q}{2}+i P\right) \phi}$ and the closed string tachyon $T=\mu e^{b \phi}$, $1 / \varepsilon$ is identified as

$$
\frac{1}{\varepsilon}=\frac{1}{b} \log \left(\frac{\Lambda}{|\mu|}\right)
$$

where $\Lambda$ is a cut-off. After removing the divergent $\log \Lambda$ term, we obtain

$$
Z\left(\sigma, 0 \mid \sigma^{\prime}, 0\right)=-\frac{1}{2} \log \mu-\log \left(2 \cosh \theta+2 \cosh \theta^{\prime}\right)+\text { const } .
$$

\section{A.2. Charged-Charged Amplitude: $Z\left(\sigma, \xi \mid \sigma^{\prime}, \xi^{\prime}\right)$}

The annulus between charged FZZT branes consists of two contributions: one from the NSNS sector and the other from the RR sector. From the explicit form of the boundary states (2.5), one can see that the NSNS sector exchange is the half of neutral brane amplitude computed in the previous section

$$
Z\left(\sigma, \xi \mid \sigma^{\prime}, \xi^{\prime}\right)_{N S}=\frac{1}{2} Z\left(\sigma, 0 \mid \sigma^{\prime}, 0\right) .
$$


On the other hand, the RR sector exchange is given by

$$
Z\left(\sigma, \xi \mid \sigma^{\prime}, \xi^{\prime}\right)_{R}=-\xi \xi^{\prime} \int_{0}^{\infty} d P\left|A_{R}(P)\right|^{2} \cos (\pi P \sigma) \cos \left(\pi P \sigma^{\prime}\right) \int_{0}^{\infty} d t_{c} \frac{\eta(q)}{f_{R}(q)} q^{\frac{P^{2}}{2}} .
$$

Here we reversed the sign of RR bra-state in order to impose the correct GSO projection in the open string channel. Again, using (A.3) and (A.4), the $t_{c}$ integral is evaluated as

$$
\int_{0}^{\infty} d t_{c} \frac{\eta(q)}{f_{R}(q)} q^{\frac{P^{2}}{2}}=\frac{1}{2 P \sinh \frac{\pi P}{\sqrt{2}}}
$$

Plugging this into (A.13), it turns out that RR exchange is also proportional to $Z\left(\sigma, 0 \mid \sigma^{\prime}, 0\right)$

$$
Z\left(\sigma, \xi \mid \sigma^{\prime}, \xi^{\prime}\right)_{R}=-\frac{\xi \xi^{\prime}}{2} Z\left(\sigma, 0 \mid \sigma^{\prime}, 0\right)
$$

Finally the total amplitude is

$$
Z\left(\sigma, \xi \mid \sigma^{\prime}, \xi^{\prime}\right)=Z\left(\sigma, \xi \mid \sigma^{\prime}, \xi^{\prime}\right)_{N S}+Z\left(\sigma, \xi \mid \sigma^{\prime}, \xi^{\prime}\right)_{R}=\frac{1-\xi \xi^{\prime}}{2} Z\left(\sigma, 0 \mid \sigma^{\prime}, 0\right) .
$$

\section{A.3. Neutral-Charged Amplitude: $Z\left(\sigma, 0 \mid \sigma^{\prime}, \xi\right)$}

The annulus between a neutral FZZT-brane and a charged FZZT-brane is given by

$$
Z\left(\sigma, 0 \mid \sigma^{\prime}, \xi\right)=-\sqrt{2} \int_{0}^{\infty} d P\left|A_{N S}(P)\right|^{2} \cos (\pi P \sigma) \cos \left(\pi P \sigma^{\prime}\right) \int_{0}^{\infty} d t_{c} \frac{\eta(q)}{f_{\widetilde{N S}}(q)} q^{\frac{P^{2}}{2}}
$$

In the same way as before, the $t_{c}$ integral is found to be

$$
\int_{0}^{\infty} d t_{c} \frac{\eta(q)}{f_{\widetilde{N S}}(q)} q^{\frac{P^{2}}{2}}=\frac{\tanh \sqrt{2} \pi P}{\sqrt{2} P}
$$

Then (A.17) becomes

$$
\begin{aligned}
Z\left(\sigma, 0 \mid \sigma^{\prime}, \xi\right) & =-\sqrt{2} \int_{-\infty}^{\infty} \frac{d P}{2}\left|A_{N S}(P)\right|^{2} \cos (\pi P \sigma) \cos \left(\pi P \sigma^{\prime}\right) \frac{\tanh \sqrt{2} \pi P}{\sqrt{2} P} \\
& =-\int_{-\infty}^{\infty} \frac{d P}{P} \frac{\cos (\pi P \sigma) \cos \left(\pi P \sigma^{\prime}\right)}{\sinh (\sqrt{8} \pi P)} \cosh \frac{\pi P}{\sqrt{2}} .
\end{aligned}
$$

Finally, regularizing the divergence using (A.8) and closing the contour of the $P$-integral, we find

$$
\begin{aligned}
& Z\left(\sigma, 0 \mid \sigma^{\prime}, \xi\right) \\
= & \frac{1}{2} \log \left(2 \sqrt{\mu} \cosh \left(\theta+\theta^{\prime}\right)+\sqrt{2 \mu}\right)+\frac{1}{2} \log \left(2 \sqrt{\mu} \cosh \left(\theta-\theta^{\prime}\right)+\sqrt{2 \mu}\right) \\
= & \frac{1}{2} \log \left[\cosh \left(\theta+\frac{\pi i}{4}\right)+\cosh \theta^{\prime}\right]+\frac{1}{2} \log \left[\cosh \left(\theta-\frac{\pi i}{4}\right)+\cosh \theta^{\prime}\right]+\log (2 \sqrt{\mu}) .
\end{aligned}
$$




\section{References}

[1] I. R. Klebanov, J. Maldacena and N. Seiberg, "Unitary and complex matrix models as 1-d type 0 strings," Commun. Math. Phys. 252, 275 (2004) arXiv:hep-th/0309168].

[2] C. V. Johnson, "Non-perturbative string equations for type 0A," JHEP 0403, 041 (2004) arXiv:hep-th/0311129.

[3] N. Seiberg and D. Shih, "Branes, rings and matrix models in minimal (super)string theory," JHEP 0402, 021 (2004) arXiv:hep-th/0312170.

[4] C. V. Johnson, "Tachyon condensation, open-closed duality, resolvents, and minimal bosonic and type 0 strings," arXiv:hep-th/0408049.

[5] D. Gaiotto, L. Rastelli and T. Takayanagi, "Minimal superstrings and loop gas models," arXiv:hep-th/0410121.

[6] A. Kapustin, "A remark on worldsheet fermions and double-scaled matrix models," arXiv:hep-th/0410268.

[7] H. Kawai, T. Kuroki and Y. Matsuo, "Universality of nonperturbative effect in type 0 string theory," arXiv:hep-th/0412004.

[8] J. E. Carlisle, C. V. Johnson and J. S. Pennington, "Baecklund transformations, Dbranes, and fluxes in minimal type 0 strings," arXiv:hep-th/0501006.

[9] J. Maldacena, G. W. Moore, N. Seiberg and D. Shih, "Exact vs. semiclassical target space of the minimal string," JHEP 0410, 020 (2004) arXiv:hep-th/0408039.

[10] A. Hashimoto, M. x. Huang, A. Klemm and D. Shih, "Open / closed string duality for topological gravity with matter," arXiv:hep-th/0501141.

[11] D. Gaiotto and L. Rastelli, "A paradigm of open/closed duality: Liouville D-branes and the Kontsevich model," arXiv:hep-th/0312196.

[12] N. Seiberg and D. Shih, "Flux vacua and branes of the minimal superstring," JHEP 0501, 055 (2005) arXiv:hep-th/0412315.

[13] T. Fukuda and K. Hosomichi, "Super Liouville theory with boundary," Nucl. Phys. B 635, 215 (2002) arXiv:hep-th/0202032.

[14] C. Ahn, C. Rim and M. Stanishkov, "Exact one-point function of N = 1 super-Liouville theory with boundary," Nucl. Phys. B 636, 497 (2002) arXiv:hep-th/0202043].

[15] E. J. Martinec, "The annular report on non-critical string theory," arXiv:hepth/0305148.

[16] D. Kutasov, K. Okuyama, J. w. Park, N. Seiberg and D. Shih, "Annulus amplitudes and ZZ branes in minimal string theory," JHEP 0408, 026 (2004) arXiv:hepth/0406030].

[17] A. Morozov, "Integrability and matrix models," Phys. Usp. 37, 1 (1994) arXiv:hepth/9303139]. 